\title{
Morphological Variation in Arabica Coffee (Coffea arabica L.) Growing in North Sumatra Indonesia
}

\author{
Variasi Morfologi Kopi Arabica (Coffea arabica L.) yang Tumbuh di Sumatera Utara Indonesia
}

\author{
Sabam Malau* and Samse Pandiangan \\ Nommensen HKBP University, Faculty of Agriculture, Department of Agroecotechnology \\ Jl. Sutomo 4-A, Medan, Indonesia
}

Received 4 January 2018/Accepted 13 August 2018

\begin{abstract}
Genetic variation is important in plant breeding. However, information on the genetic variability of Arabica coffee especially in coffee field of North Sumatra was not yet available. Magnitude of morphological variation, genotypic variation, phenotypic variation, heritability, genetic advance, genetic correlation, and phenotypic correlation of plant vigors and yield components of 28 genotypes were evaluated using nested design. This research showed morphological and genetic variations of the genotypes in the field. Based on the research locations as operational taxonomic unit, the genotypes were separated into three clusters. Most of the parameters had low to moderate genotypic variation, while phenotypic variation was moderate to high. Heritability and genetic advance were low, moderate, and high. Several plant vigors and yield components had a positive significant genetic and phenotypic correlation one another, and several had negative ones. Coffee berry borer infestation (CBBI) had a highly significant negative genetic correlation with leaf width ( $\left.r_{G}=-0.309 * *\right)$, leaf weight ( $r_{G}$ $\left.=-0.671^{* *}\right)$, fruit diameter $\left(r_{G}=-0.320^{* *}\right)$, and bean length $\left(r_{G}=-0.175^{* *}\right)$. CBBI showed a significant positive genetic correlation with mesocarp $\mathrm{pH}\left(r_{G}=0.134 *\right)$. To reduce CBBI, selection for higher leaf weight is better. Selection on lower pH of mesocarp could be considered to decrease CBBI.
\end{abstract}

Keywords: cluster analysis, genetic correlation, genetic heritability, variability

\section{ABSTRAK}

Variasi genetik merupakan dasar bagi pemuliaan tanaman. Akan tetapi, informasi tentang variabilitas genetik kopi Arabica yang ditemukan di ladang kopi di Sumatera Utara belum tersedia. Variasi morfologi, variasi genotipik, variasi fenotipik, heritabilitas, kemajuan genetik, korelasi genetik, dan korelasi fenotipik dari vigor tanaman dan komponen produksi dari 28 genotipe kopi Arabica diteliti dengan menggunakan rancangan tersarang. Penelitian ini menunjukkan variasi morfologis dan genetik dari genotipe. Berdasarkan lokasi penelitian sebagai unit taksonomi operasional, genotipe menyebar ke dalam tiga kluster. Hampir semua parameter mempunyai variasi genetik yang rendah hingga sedang, sedangkan variasi fenotipik sedang hingga tinggi. Heritabilitas dan kemajuan genetik rendah, sedang dan tinggi. Beberapa vigor tanaman dan komponen produksi mempunyai korelasi genetik dan fenotipik yang positif dan signifikan satu dengan lainnya, sedangkan beberapa lainnya memiliki korelasi yang negatif. Infestasi penggerek buah kopi (CBBI) menunjukkan korelasi genetik yang sangat signifikan dan negatif dengan lebar daun $\left(r_{G}=-0.309 * *\right)$, bobot daun $\left(r_{G}=-0.671 * *\right)$, diameter buah $\left(r_{G}=-0.320 * *\right)$, dan panjang biji $\left(r_{G}=-0.175^{*}\right)$. CBBI mempunyai korelasi genetik yang signifikan dan positif dengan pH daging buah $\left(r_{G}=0.134 *\right)$. Untuk mengurangi CBBI, lebih baik memilih tanaman dengan bobot daun yang berat. Tanaman dengan pH daging buah yang rendah dapat dipilih untuk mengurangi $C B B I$.

Kata kunci: analisis kluster, heritabilitas, keragaman genetik, korelasi genetik

\section{INTRODUCTION}

Arabica coffee (Coffea arabica L.) was cultivated for the first time around 120 years ago in North Sumatra. In Indonesia, North Sumatra is one of the most important production center of Arabica coffee. Indonesia produced

\footnotetext{
* Corresponding author. e-mail: drsabammalau@gmail.com
}

189,834 tons of Arabica coffee beans in 2016, of which North Sumatra contributed 53,237 tons green beans per year (DGEC, 2017). A total of 63,339 ha of Arabica coffee growing areas are located in North Sumatra, which become source of livelihood for 143,061 coffee farmers. In recent years, Arabica coffee cultivation is facing climate change (Sudradjat, 2010) which can be seen at coffee plantation in nine districts of North Sumatra with the altitude between 800 to $1,600 \mathrm{~m}$ above sea level (asl). This environmental 
pressures might create genetic mutation lead to genetic variation.

Although Indonesian Goverment has released several commercial cultivars, empirical facts showed that many of coffee farmers are still using traditional seeds from unknown resources for their new cultivation field which might cause low coffee productivity (1.14 ton $\mathrm{ha}^{-1}$ of green bean), and might cause genetic variation among farmers' land. Low productivity could also be affected by soil fertility (Hanisch et al., 2011) and coffee berry borer (CBB) attact which is considered as one of the most destructive pest of Arabica coffee in North Sumatra.

Plant breeders require genetic variability of desirable characters to carry out the breeding programs (Mayo, 1987; Mishra and Slater, 2012; Constantin et al., 2017). Genetic variation of Arabica coffee can be found not only in cultivated cultivars (Setotaw et al., 2010; Tessema et al., 2011; Geleta et al., 2012; Fatimah et al., 2014; Randriani et al., 2014; Dani et al., 2016), but also in wild populations (Schmitt et al., 2009; Aerts et al., 2013; Atinafu et al., 2017). Previous studies done by Silvestrini et al. (2008) and Kathurima et al. (2012) exhibited that genetic variation in commercial coffee cultivar was narrow. However, another study conducted by Geleta et al. (2012) revealed broad genetic variation in the collection of Arabica coffee cultivars. Genetic diversity was shown to be correlated with morphological diversity (Yuan et al., 2015). However, information on genetic diversity of Arabica coffee derived from North Sumatra was not yet available. The aim of this research was to determine morphological and genetic variations of Arabica coffee at coffee plantations in North Sumatra.

\section{MATERIALS AND METHODS}

The research was carried out in District Tapanuli Utara, Toba Samosir, Humbang Hasundutan, Samosir, Simalungun, Pakpak Bharat and Dairi, North Sumatra Province. Data was collected in July 2014. The nested design with three factors was used for data analysis (Quinn and Keough, 2002). The first step was to select 7 districts, then 2 sub-districts were chosen in each district, and the final step was to select 2 coffee farms in each sub-district. These selected coffee farms were treated as genotypes $(\mathrm{G})$. Each farm consisted of 200-300 plants of variety Sigarar Utang which is Arabica coffee. The plants were 6-7 years old, with the characteristics of having a shot of bronze-colored leaves, ripe fruits, and harvest frequency of once in two weeks. Ten plants were selected randomly in each farm. In total, twenty eight genotypes of Arabica coffee were used to determine morphological and genetic variation in this study. Mesocarp $\mathrm{pH}$ was measured using $\mathrm{pH}$ meter (Amtast KS-05 vergara). A fruit showing the frass on the entrance hole is a $\mathrm{CBB}$ infected fruit which is caused by females of $\mathrm{CBB}$ live inside the fruit after boring a hole at dictus or near the dictus (Vega et al., 2009). All fruits were checked. Coffee berry borer infestation (CBBI) was the ratio of the number of infected fruits to the total number of fruits (\%).

Tree morphology comprises plant vigor (plant height, leaf length, leaf width, leaf weight), yield components (100 fruits weight, fruit length, fruit diameter, mesocarp thickness, mesocarp $\mathrm{pH}, 100$ parchments weight, parchment length, parchment width, parchment thickness, 100 beans weight, bean length, bean width, bean thickness), and coffee berry borer infestation (CBBI) (Wahyudi et al., 2016). All data were analyzed with the hierarchical cluster analysis using nearest neighbour cluster method measured with squared Euclidean distance. In the analysis, research location was used as operational taxonomic unit (OTU) while coffee morphology were treated as variables.

The additive effect model for the nested design with three factors was $\mathrm{Y}_{\mathrm{ijkl}}=\mu+\mathrm{D}_{\mathrm{i}}+\mathrm{S}_{\mathrm{j}(\mathrm{i})}+\mathrm{G}_{\mathrm{k}(\mathrm{j}(\mathrm{i}))}+\mathrm{E}_{\mathrm{l}(\mathrm{k}(\mathrm{j}(\mathrm{i})))}$ where $\mathrm{Y}_{\mathrm{ijkl}}=i j k l$ th observation, $\mu=$ general mean, $\mathrm{D}_{\mathrm{i}}=$ effect for $i$ th districts, $\mathrm{S}_{\mathrm{j}(\mathrm{i})}=$ effect for $j$ th sub-districs within $i$ th districts, $\mathrm{G}_{\mathrm{k}(\mathrm{j}(\mathrm{i}))}=$ effect for $k$ th genotype within $j$ th sub-districts within $i$ th districts, and $\mathrm{E}_{1(\mathrm{k}(\mathrm{j}(\mathrm{i})))}=$ error (Quinn and Keough, 2002). Estimated variance component (EVC) for phenotype $=\mathrm{s}_{\mathrm{P}}^{2}=\mathrm{s}_{\mathrm{G}}^{2}+\mathrm{s}_{\mathrm{E}}^{2}$ (Table 1). Genotypic coefficient of variation $(\mathrm{GCV})=\left(\left(\mathrm{s}_{\mathrm{G}}^{2}\right)^{0.5} / \mathrm{m}\right) \times 100 \%$, and phenotypic coefficient of variation $(\mathrm{PCV})=\left(\left(\mathrm{s}_{\mathrm{P}}^{2}\right)^{0.5} / \mathrm{m}\right) \times 100 \%$ where $\mathrm{m}=$ mean of phenotype (Mayo, 1987). Coefficient of heritability in broad sense $\left(\mathrm{H}^{2}{ }_{\mathrm{bs}}\right)=\mathrm{H}^{2}{ }_{\mathrm{bs}}=\mathrm{s}_{\mathrm{G}}{ }_{\mathrm{G}} / \mathrm{s}_{\mathrm{P}}{ }^{2}$. Estimated genetic advance $(\mathrm{GA})=\mathrm{i} \times\left(\mathrm{s}_{\mathrm{p}}^{2}\right)^{0.5} \mathrm{x} \mathrm{H}^{2}{ }_{\mathrm{bs}}$. Then, GAM as expression of GA in percentage of mean $(\mathrm{m})=(\mathrm{GA} / \mathrm{m}) \times 100 \%$ where $\mathrm{i}$ $=2.063$ at selection intensity $5 \%$ (Mayo, 1987). GCV, PCV and GAM were stated as low $(<5 \%)$, moderate $(5-10 \%)$, and high $(>10 \%) . \mathrm{H}^{2}$ bs was defined as low $(40 \%)$, moderate (40$60 \%$ ), and high $(>60 \%)$. Genetic correlation coefficient $r_{G x y}$ between two phenotypes ( $x$ and $y)=r_{G x y}=\operatorname{cov}_{G(x y)} /\left(\sigma_{G x}^{2} x\right.$ $\left.\sigma^{2}{ }_{G y}\right)^{0.5}$ while phenotypic correlation coefficient $r_{P x y}$ between two $=\operatorname{cov}_{\mathrm{p}(\mathrm{xy})} /\left(\sigma_{\mathrm{Px}}^{2} \mathrm{x} \sigma_{\mathrm{Py}}^{2}\right)^{0.5}$ whereby $\operatorname{cov}_{\mathrm{Gxy}}$ was genetic covariance between phenotypes $\mathrm{x}$ and $\mathrm{y}$, and $\operatorname{cov}_{\text {Pxy }}$ was phenotypic covariance between phenotypes $\mathrm{x}$ and y (Mayo, 1987). The significance of the correlation coefficients $r_{G x y}$ and $r_{\text {Pxy }}$ was compared to critical $r$ tabular value at $\alpha=0.05$ and $\alpha=0.01$ using the degree of freedom of the error (Quinn and Keough, 2002). IBM SPSS version 19 and Microsoft Excel version 2007 was used for data analysis.

\section{RESULTS AND DISCUSSION}

The cluster dendrogram showed morphological variation among research locations (Figure 1). Gichimu and Omondi (2010) found the correlation between morphological variation with genetic variation of coffee genotypes. Genotypes were significantly different in plant vigor, yield components, and CBBI (Table 2).

This research found low and moderate genetic variation in several plant vigor and yield components while high one in CBBI (Table 3). The results of this research might be generally in line with Kitila et al. (2011) and Beksisa and Ayano (2016) who found low, moderate and high genetic variation in fruit length and fruit diameter, plant height, bean length, and bean width. Tessema et al. (2011) found the similar result in bean weight but Kitila et al. (2011) revealed high genetic variation in bean weight. Low and moderate genotypic variation in most of the parameters might indicate the nature of self-fertilized coffee plants. Broad genetic variability must be obtained through hybridization. 


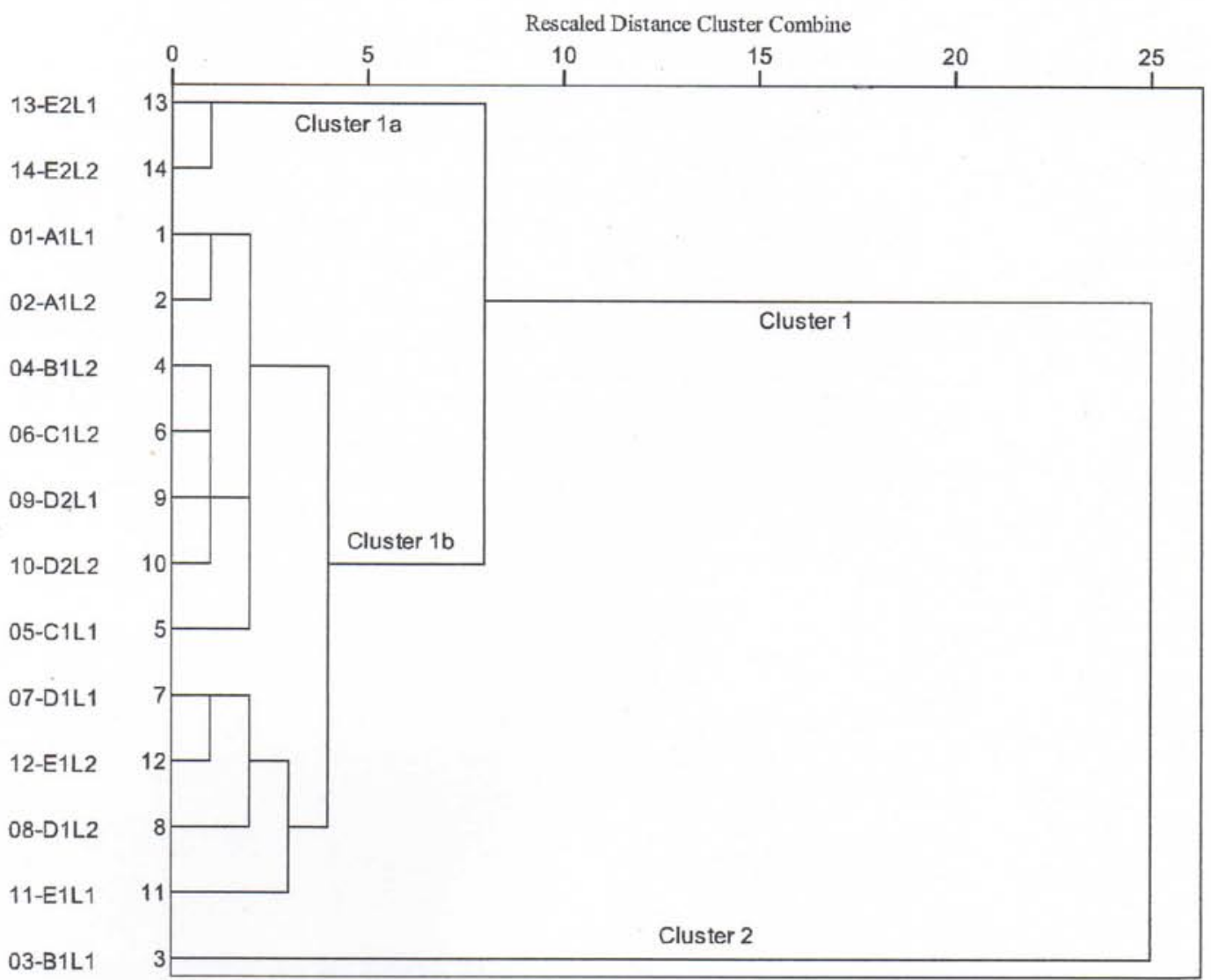

Figure 1. Cluster analysis based on 14 locations using 18 morphological variables of the genotypes of Arabica coffee

Note: 01-A1L1 = Location 1 (Sub-district Parlilitan 1 in District Humbanghas), 02-A1L2 = Location 2 (Sub-district Parlilitan 2 in District Humbanghas), 03-B1L1 = Location 3 (Sub-district Dolok Pangaribuan in District Simalungun), 04-B1L2 = Location 4 (Sub-district Tanjung Dolok in District Simalungun), 05-C1L1 = Location 5 (Sub-district Kerajaan in District Pakpak Bharat), 06-C1L2=Location 6 (Sub-district Tinada in District Pakpak Bharat), 07-D1L1 = Location 7 (Sub-district Pangururan in District Samosir), 08-D1L2 = Location 8 (Sub-district Ronggur Nihuta in District Samosir), 09-D2L1 = Location 9 (Sub-district Parbuluan 1 in District Dairi), 10-D2L2 = Location 10 (Sub-district Parbuluan 2 in District Dairi), 11-E1L1 = Location 11 (Sub-district Siborong-borong in District North Tapanuli), 12-E1L2 = Location 12 (Sub-district Sipaholon in District North Tapanuli), 13-E2L1 = Location 13 (Sub-district Uluan in District Tobasa), 14-E2L2 = Location 14 (Sub-district Sigumpar in Tobasa)

Plant vigor and yield components showed moderate to high phenotypic variation (Table 3 ). The results of this research supported Kittila et al. (2011) and Beksisa and Ayano (2016) who found moderate to high phenotypic variation in plant height, fruit diameter, bean length and bean width. In contrary to this research, low phenotypic variation in fruit length and high phenotypic variation in bean weight were found by Kitila et al. (2011) and Tessema et al. (2011).

This research showed low, moderate, and high heritability in several plant vigor component and yield components (Table 3 ). High heritability was manifested by leaf weight, fruit weight, mesocarp $\mathrm{pH}$, parchment weight, and CBBI. These research results were in line with Kitila et al. (2011) who found high heritability in plant height, fruit length, fruit diameter, bean weight, bean length and bean width. However, Kitila et al. (2011) found high heritability in plant height, fruit length, fruit diameter, bean weight, bean length and bean width while Bekisa and Ayono (2016) revealed low heritability in plant height, and Tessema et al. (2011) found high heritability in bean weight.
This research revealed low to high genetic advance in several plant vigor components and yield components (Table 3). In contrary to this result, Kitila et al. (2011) and Bekisa and Ayono (2016) found moderate genetic advance in several plant vigor and yield components. Kitila et al. (2011) and Tessema et al. (2011) found high genetic advance bean weight. Two-thirds $(66.7 \%)$ of the parameters had low to moderate genetic advance. This might relate to narrow genotypic variation of the existing coffee cultivars as selffertilized coffee plants.

This research found that all plant vigor components (plan height, leaf length, leaf width, leaf weight) had a high significant genetic correlation (Table 4). Genetic correlation between several vigor parameters one another and with yield components was also found by Kitila et al. (2011). Selection for leaf weight would be the first priority to increase resistance of plant against CBB. The selection could be possible to be carried out successfully due to high heritability. The lower $\mathrm{pH}$ of mesocarp was the less CBBI was. Consequently, selection for lower $\mathrm{pH}$ of mesocarp could decrease CBBI. The selection could be conducted 
Table 1. Estimation of variance analysis for nested design with factors district $(p=7$ levels), subdistrict within district $(q=$ 2 levels) and genotype within subdistrict within district $(\mathrm{r}=2$ levels $)$ and sample $(\mathrm{n}=10)$

\begin{tabular}{|c|c|c|c|c|c|}
\hline Source of variation & $\mathrm{df}$ & $\mathrm{MS}$ & F-ratio & EMS & $\mathrm{EVC}$ \\
\hline District (D) & $\mathrm{p}-1$ & $\mathrm{MS}_{\mathrm{D}}$ & $\mathrm{MS}_{\mathrm{D}} / \mathrm{MS}_{\mathrm{S}(\mathrm{D})}$ & $\begin{array}{l}\sigma_{E}^{2}+\mathrm{n} \sigma^{2} \mathrm{G}+\mathrm{nr} \sigma_{S}^{2} \\
+\mathrm{nrq} \sigma_{D}^{2}\end{array}$ & $\mathrm{~s}_{\mathrm{D}}^{2}=\left(\mathrm{MS}_{\mathrm{D}}-\mathrm{MS}_{\mathrm{S}(\mathrm{D}))} / \mathrm{nrq}\right.$ \\
\hline $\begin{array}{l}\text { Subdistrict nested in District } \\
\text { (S(D)) }\end{array}$ & $\mathrm{p}(\mathrm{q}-1)$ & $\mathrm{MS}_{\mathrm{S}(\mathrm{D})}$ & $\mathrm{MS}_{\mathrm{S}(\mathrm{D})} / \mathrm{MS}_{\mathrm{G}(\mathrm{S}(\mathrm{D}))}$ & $\sigma_{E}^{2}+\mathrm{n} \sigma_{G}^{2}+\mathrm{nr} \sigma_{S}^{2}$ & $\mathrm{~s}_{\mathrm{S}}^{2}=\left(\mathrm{MS}_{\mathrm{S}(\mathrm{D})}-\mathrm{MS}_{\mathrm{G}(\mathrm{S}(\mathrm{D}))} / \mathrm{nr}\right.$ \\
\hline $\begin{array}{l}\text { Genotype nested subdistrict } \\
\text { nested in district }(\mathrm{G}(\mathrm{S}(\mathrm{D})))\end{array}$ & $\mathrm{pq}(\mathrm{r}-1)$ & $\mathrm{MS}_{\mathrm{G}(\mathrm{S}(\mathrm{D})}$ & $\mathrm{MS}_{\mathrm{G}(\mathrm{S}(\mathrm{D}))} / \mathrm{MS}_{\mathrm{Error}}$ & $\sigma_{E}^{2}+\mathrm{n} \sigma_{G}^{2}$ & $\mathrm{~s}_{\mathrm{G}}^{2}=\left(\mathrm{MS}_{\mathrm{G}(\mathrm{S}(\mathrm{D}))}-\mathrm{MS}_{\text {Error })} / \mathrm{n}\right.$ \\
\hline Residual & $\operatorname{pqr}(\mathrm{n}-1)$ & $\mathrm{MS}_{\text {Error }}$ & & $\sigma_{E}^{2}$ & $\mathrm{~s}_{E}^{2}=\mathrm{MS}_{\text {Error }}$ \\
\hline
\end{tabular}

Note: $\mathrm{df}=$ degree of freedom, $\mathrm{MS}=$ mean square, $\mathrm{EMS}=$ expected mean square, $\mathrm{EVC}=$ estimated variance component, $\mathrm{s}_{\mathrm{D}}^{2}=\mathrm{EVC}$ for districts, $\mathrm{s}_{\mathrm{S}}^{2}=\mathrm{EVC}$ for sub-districts, $\mathrm{s}_{\mathrm{G}}^{2}=\mathrm{EVC}$ for genotypes, $\mathrm{s}_{E}^{2}=\mathrm{EVC}$ for error $=\mathrm{MS}_{\text {Error }}$. Hence, EVC for phenotype $=\mathrm{s}_{\mathrm{P}}^{2}=\mathrm{s}_{\mathrm{G}}^{2}$ $+\mathrm{s}^{2}$

Table 2. Analysis of variance of district, subdistrict, genotype and estimated variance components of parameters

\begin{tabular}{|c|c|c|c|c|c|c|c|c|c|c|c|c|}
\hline & $\begin{array}{c}\text { MS } \\
\text { district } \\
(\mathrm{p}=7 ; \\
\mathrm{df}=6)\end{array}$ & $\begin{array}{c}\text { MS } \\
\text { subdistrict } \\
\begin{aligned}(\mathrm{q}=2 ; \mathrm{df} \\
=7)\end{aligned}\end{array}$ & $\begin{array}{c}\text { MS } \\
\text { genotype } \\
\begin{array}{c}(\mathrm{r}=2 ; \mathrm{df} \\
=14)\end{array}\end{array}$ & $\begin{array}{l}\text { MS } \\
\text { error } \\
(\mathrm{df}= \\
252)\end{array}$ & $\begin{array}{l}\text { F-ratio } \\
\text { for } \\
\text { district }\end{array}$ & $\begin{array}{l}\text { F-ratio for } \\
\text { subdistrict }\end{array}$ & $\begin{array}{c}\text { F-ratio for } \\
\text { genotype }\end{array}$ & $\mathrm{S}^{2}{ }_{\mathrm{D}}$ & $\mathrm{s}_{\mathrm{S}}^{2}$ & $\mathrm{~s}^{2}{ }_{\mathrm{G}}$ & $\mathrm{s}_{\mathrm{E}}^{2}$ & $\mathrm{~S}_{\mathrm{P}}^{2}$ \\
\hline $\mathrm{PH}$ & 0.05 & 0.04 & 0.04 & 0.01 & $1.23 \mathrm{~ns}$ & $1.08 \mathrm{~ns}$ & $3.91 * *$ & 0.000 & 0.000 & 0.003 & 0.01 & 0.01 \\
\hline LL & 50.05 & 11.52 & 11.42 & 0.72 & $4.35^{*}$ & $1.01 \mathrm{~ns}$ & $15.96 * *$ & 0.96 & 0.01 & 1.07 & 0.72 & 1.79 \\
\hline LWi & 4.20 & 2.17 & 1.94 & 0.20 & $1.94 \mathrm{~ns}$ & $1.12 \mathrm{~ns}$ & $9.91 * *$ & 0.05 & 0.01 & 0.17 & 0.20 & 0.37 \\
\hline LWe & 1.49 & 0.27 & 0.15 & 0.01 & $5.44 *$ & $1.88 \mathrm{~ns}$ & $16.44 * *$ & 0.03 & 0.01 & 0.01 & 0.01 & 0.02 \\
\hline HFW & $6,888.10$ & $1,101.50$ & 786.30 & 48.08 & $6.25 *$ & $1.40 \mathrm{~ns}$ & $16.35^{* *}$ & 144.70 & 15.76 & 73.82 & 48.08 & 121.90 \\
\hline FL & 0.80 & 0.09 & 0.08 & 0.02 & $9.00 * *$ & $1.10 \mathrm{~ns}$ & $3.26 * *$ & 0.02 & 0.000 & 0.01 & 0.02 & 0.03 \\
\hline FD & 0.11 & 0.02 & 0.02 & 0.01 & $5.22 *$ & $1.01 \mathrm{~ns}$ & $2.20 * *$ & 0.002 & 0.000 & 0.001 & 0.01 & 0.01 \\
\hline MT & 0.90 & 0.19 & 0.11 & 0.03 & $4.70^{*}$ & $1.81 \mathrm{~ns}$ & $3.26^{* *}$ & 0.02 & 0.004 & 0.01 & 0.03 & 0.04 \\
\hline $\mathrm{MpH}$ & 3.14 & 2.25 & 1.92 & 0.09 & $1.39 \mathrm{~ns}$ & $1.17 \mathrm{~ns}$ & $21.55^{* *}$ & 0.02 & 0.02 & 0.18 & 0.09 & 0.27 \\
\hline HPW & 814.8 & 167.20 & 155.70 & 8.22 & $4.87^{*}$ & $1.07 \mathrm{~ns}$ & $18.95 * *$ & 16.19 & 0.57 & 14.75 & 8.22 & 22.97 \\
\hline PL & 0.19 & 0.03 & 0.02 & 0.004 & $6.12 *$ & $1.47 \mathrm{~ns}$ & $5.30 * *$ & 0.004 & 0.001 & 0.002 & 0.004 & 0.01 \\
\hline PWi & 0.16 & 0.02 & 0.004 & 0.003 & $8.30 * *$ & $4.33 * *$ & $1.43 \mathrm{~ns}$ & 0.003 & 0.001 & 0.000 & 0.003 & 0.003 \\
\hline PT & 0.02 & 0.01 & 0.01 & 0.003 & $4.47^{*}$ & $1.02 \mathrm{~ns}$ & $1.84 *$ & 0.001 & 0.000 & 0.000 & 0.003 & 0.003 \\
\hline HBW & 38.77 & 9.16 & 4.70 & 0.80 & $4.23 *$ & $1.95 \mathrm{~ns}$ & $5.84 * *$ & 0.74 & 0.22 & 0.39 & 0.80 & 1.19 \\
\hline $\mathrm{BL}$ & 0.04 & 0.01 & 0.01 & 0.003 & $2.79 \mathrm{~ns}$ & $1.44 \mathrm{~ns}$ & $3.35 * *$ & 0.001 & 0.000 & 0.001 & 0.003 & 0.003 \\
\hline BWi & 0.01 & 0.003 & 0.003 & 0.001 & $3.74 \mathrm{~ns}$ & $1.03 \mathrm{~ns}$ & $3.00 * *$ & 0.000 & 0.000 & 0.000 & 0.001 & 0.001 \\
\hline BT & 0.01 & 0.002 & 0.001 & 0.001 & $3.40 \mathrm{~ns}$ & $1.94 \mathrm{~ns}$ & $1.16 \mathrm{~ns}$ & 0.000 & 0.000 & 0.000 & 0.001 & 0.001 \\
\hline CBBI & $6,146.50$ & $1,077.80$ & $1,011.70$ & 16.31 & $5.70^{*}$ & $1.07 \mathrm{~ns}$ & $62.03 * *$ & 126.70 & 3.30 & 99.54 & 16.31 & 115.90 \\
\hline
\end{tabular}

Note: $\mathrm{PH}=$ plant height $(\mathrm{m}), \mathrm{LL}=$ leaf length $(\mathrm{cm}), \mathrm{LWi}=$ leaf width $(\mathrm{cm}), \mathrm{LWe}=$ leaf weight $(\mathrm{g}), \mathrm{HFW}=100$ fruits weight $(\mathrm{g}), \mathrm{FL}=$ fruit length $(\mathrm{cm}), \mathrm{FD}=$ fruit diameter $(\mathrm{cm}), \mathrm{MT}=$ mesocarp thickness $(\mathrm{cm}), \mathrm{MpH}=$ mesocarp $\mathrm{pH}, \mathrm{HPW}=100$ parchments weight $(\mathrm{g}), \mathrm{PL}$ $=$ parchment length $(\mathrm{cm}), \mathrm{PWi}=$ parchment width $(\mathrm{cm}), \mathrm{PT}=$ parchment thickness $(\mathrm{cm}), \mathrm{HBW}=100$ beans weight $(\mathrm{g}), \mathrm{BL}=\mathrm{bean}$ length $(\mathrm{cm}), \mathrm{BWi}=$ bean width $(\mathrm{cm}), \mathrm{BT}=$ bean thickness $(\mathrm{cm}), \mathrm{CBBI}=$ coffee berry borer infestation $(\%)$. For districts, F-table at $\alpha 0.05=3.87$ and at $\alpha 0.01=7.19$. For sub-districts, F-table at $\alpha 0.05=2.77$, and at $\alpha 0.01=4.28$. For genotypes, F-table at $\alpha 0.05$ $=1.73$ and at $\alpha 0.01=2.15, \mathrm{~ns}=$ not significant, $*=$ significant at $\alpha 0.05, * *=$ highly significant at $\alpha 0.01$

successfully due to moderate genetic variation. This selection may be combined with selection for yield in the first high-yield year (Oliveira et al., 2010).

This research revealed that several plant vigor and yield components phenotypically correlated each others (Table 4). Kitila et al. (2011), Rodrigues et al. (2012), and Gessese et al. (2015) found a phenotypic correlation between several plant vigor parameters and yield components.

In the future research, it would be necessary to examine how $\mathrm{pH}$ of mesocarp could affect CBBI. Lower $\mathrm{pH}$ might cause an unpleasant taste for $\mathrm{CBB}$. Lower $\mathrm{pH}$ might affect certain chemical substances in coffee fruit so that the pest 
Table 3. Genetic components of parameters

\begin{tabular}{lrrrrrrrrrr}
\hline Parameter & Minimum & Maximum & \multicolumn{1}{c}{ Mean } & \multicolumn{1}{c}{$\mathrm{s}_{\mathrm{d}}$} & $\mathrm{GCV}(\%)$ & $\mathrm{PCV}(\%)$ & $\mathrm{H}_{\text {bs }}^{2}(\%)$ & $\mathrm{GA}$ & $\mathrm{GAM}(\%)$ \\
\hline PH & 1.41 & 1.72 & 1.63 & 0.03 & 3.3 & 7.0 & 22.6 & 0.05 & 3.3 \\
LL & 10.73 & 16.32 & 14.40 & 0.27 & 7.2 & 9.3 & 59.9 & 1.65 & 11.5 \\
LWi & 4.54 & 6.54 & 5.69 & 0.14 & 7.4 & 10.7 & 47.1 & 0.59 & 10.4 \\
LWe & 1.16 & 1.87 & 1.57 & 0.03 & 7.4 & 9.6 & 60.7 & 0.19 & 12.0 \\
HFW & 141.24 & 201.19 & 166.40 & 2.19 & 5.2 & 6.6 & 60.6 & 13.79 & 8.3 \\
FL & 1.34 & 1.96 & 1.63 & 0.05 & 4.6 & 10.7 & 18.4 & 0.07 & 4.1 \\
FD & 1.17 & 1.44 & 1.31 & 0.03 & 2.6 & 7.9 & 10.8 & 0.02 & 1.8 \\
MT & 0.75 & 1.52 & 1.15 & 0.06 & 7.5 & 17.4 & 18.4 & 0.08 & 6.6 \\
MpH & 4.19 & 5.42 & 4.80 & 0.09 & 8.9 & 10.9 & 67.3 & 0.72 & 15.1 \\
HPW & 40.65 & 59.87 & 50.53 & 0.91 & 7.6 & 9.5 & 64.2 & 6.35 & 12.6 \\
PL & 1.19 & 1.45 & 1.30 & 0.02 & 3.2 & 5.8 & 30.1 & 0.05 & 3.6 \\
PWi & 0.81 & 1.08 & 0.87 & 0.02 & 1.3 & 6.5 & 4.2 & 0.00 & 0.6 \\
PT & 0.59 & 0.61 & 0.57 & 0.02 & 2.8 & 9.9 & 7.7 & 0.01 & 1.6 \\
HBW & 13.81 & 14.38 & 13.96 & 0.28 & 4.5 & 7.8 & 32.6 & 0.74 & 5.3 \\
BL & 0.85 & 1.04 & 0.94 & 0.02 & 2.7 & 6.2 & 19.0 & 0.02 & 2.5 \\
BWi & 0.66 & 0.77 & 0.70 & 0.01 & 2.1 & 5.1 & 16.7 & 0.01 & 1.8 \\
BT & 0.34 & 0.39 & 0.37 & 0.01 & 1.0 & 8.0 & 1.6 & 0.001 & 0.3 \\
CBBI & 0.31 & 61.87 & 17.33 & 1.28 & 57.6 & 62.1 & 85.9 & 19.08 & 110.1 \\
\hline
\end{tabular}

Note: $\mathrm{PH}=$ plant height $(\mathrm{m}), \mathrm{LL}=$ leaf length $(\mathrm{cm}), \mathrm{LWi}=$ leaf width $(\mathrm{cm}), \mathrm{LWe}=$ leaf weight $(\mathrm{g}), \mathrm{HFW}=100$ fruits weight $(\mathrm{g}), \mathrm{FL}=$ fruit length $(\mathrm{cm}), \mathrm{FD}=$ fruit diameter $(\mathrm{cm}), \mathrm{MT}=$ mesocarp thickness $(\mathrm{cm}), \mathrm{MpH}=$ mesocarp $\mathrm{pH}, \mathrm{HPW}=100$ parchments weight $(\mathrm{g})$, PL $=$ parchment length $(\mathrm{cm}), \mathrm{PWi}=$ parchment width $(\mathrm{cm}), \mathrm{PT}=$ parchment thickness $(\mathrm{cm}), \mathrm{HBW}=100$ beans weight $(\mathrm{g}), \mathrm{BL}=$ bean length $(\mathrm{cm}), \mathrm{BWi}=$ bean width $(\mathrm{cm}), \mathrm{BT}=$ bean thickness $(\mathrm{cm}), \mathrm{CBBI}=$ coffee berry borer infestation $(\%)$, sd = standard deviation, $\mathrm{GCV}=$ genotypic coefficient of variation, $\mathrm{PCV}=$ phenotypic coefficient of variation, $\mathrm{H}_{\mathrm{bs}}^{2}=$ coefficient of heritability in broad sense, $\mathrm{GA}=$ genetic advance, $\mathrm{GAM}=$ genetic advance in percentage of mean

would stop drilling the fruit of coffee. Coffee genotypes and species might be different in chemistry whereby some of the chemotypes were shown to be insecticidal (Green et al., 2015). It would be also important to examine whether this pest could adapt to lower $\mathrm{pH}$. This pest could evolve to high caffeine content so that caffeine was no longer toxic to this pest (Filho and Mazzafera, 2003).

Table 4. Genetic $\left(\mathrm{r}_{\mathrm{G}}\right)$ and phenotypic $\left(\mathrm{r}_{\mathrm{P}}\right)$ correlation coefficient

\begin{tabular}{|c|c|c|c|c|c|c|c|c|c|c|c|c|c|c|c|c|c|c|}
\hline & & LL & LWi & LWe & HFW & FL & FD & MT & $\mathrm{MpH}$ & HPW & PL & PWi & PT & HBW & BL & BWi & BT & CBBI \\
\hline PH & $\mathrm{rG}$ & $\begin{array}{c}-0.180 \\
* *\end{array}$ & $\begin{array}{c}-0.322 \\
* *\end{array}$ & $\begin{array}{c}-0.260 \\
* *\end{array}$ & $\begin{array}{c}0.439 \\
* *\end{array}$ & $\begin{array}{c}0.064 \\
\mathrm{~ns}\end{array}$ & $\begin{array}{c}-0.564 \\
* *\end{array}$ & $\begin{array}{c}0.824 \\
* *\end{array}$ & $\begin{array}{c}0.296 \\
* *\end{array}$ & $\begin{array}{c}0.506 \\
* *\end{array}$ & $\begin{array}{c}-0.148 \\
*\end{array}$ & $\begin{array}{c}0.320 \\
* *\end{array}$ & $\begin{array}{c}0.521 \\
* *\end{array}$ & $\begin{array}{c}0.475 \\
* *\end{array}$ & $\begin{array}{c}0.324 \\
* *\end{array}$ & $\begin{array}{c}0.363 \\
* *\end{array}$ & $\begin{array}{c}0.189 \\
* *\end{array}$ & $\begin{array}{c}0.327 \\
* *\end{array}$ \\
\hline PH & $\mathrm{rP}$ & $\begin{array}{c}-0.051 \\
\mathrm{~ns}\end{array}$ & $\begin{array}{c}-0.109 \\
n s\end{array}$ & $\begin{array}{c}-0.089 \\
n s\end{array}$ & $\begin{array}{c}0.146 \\
*\end{array}$ & $\begin{array}{c}0.064 \\
\text { ns }\end{array}$ & $\begin{array}{c}0.032 \\
n s\end{array}$ & $\begin{array}{c}0.248 \\
* *\end{array}$ & $\begin{array}{c}0.049 \\
n s\end{array}$ & $\begin{array}{c}0.211 \\
* *\end{array}$ & $\begin{array}{c}0.015 \\
\text { ns }\end{array}$ & $\begin{array}{c}0.017 \\
\text { ns }\end{array}$ & $\begin{array}{c}0.030 \\
\text { ns }\end{array}$ & $\begin{array}{c}0.149 \\
* *\end{array}$ & $\begin{array}{c}0.058 \\
\mathrm{~ns}\end{array}$ & $\begin{array}{c}0.127 \\
*\end{array}$ & $\begin{array}{c}0.065 \\
\text { ns }\end{array}$ & $\begin{array}{c}0.132 \\
*\end{array}$ \\
\hline LL & $\mathrm{rG}$ & 1 & $\begin{array}{c}0.868 \\
* *\end{array}$ & $\begin{array}{c}0.284 \\
* *\end{array}$ & $\begin{array}{c}0.188 \\
* *\end{array}$ & $\begin{array}{c}0.901 \\
* *\end{array}$ & $\begin{array}{c}0.310 \\
* *\end{array}$ & $\begin{array}{c}0.040 \\
\mathrm{~ns}\end{array}$ & $\begin{array}{c}0.110 \\
n s\end{array}$ & $\begin{array}{c}0.346 \\
* *\end{array}$ & $\begin{array}{c}0.146 \\
*\end{array}$ & $\begin{array}{c}0.900 \\
* *\end{array}$ & $\begin{array}{c}0.175 \\
* *\end{array}$ & $\begin{array}{c}0.224 \\
* *\end{array}$ & $\begin{array}{c}0.163 \\
* *\end{array}$ & $\begin{array}{c}0.205 \\
* *\end{array}$ & $\begin{array}{c}0.339 \\
* *\end{array}$ & $\begin{array}{c}-0.017 \\
\text { ns }\end{array}$ \\
\hline LL & $\mathrm{rP}$ & 1 & $\begin{array}{c}0.590 \\
* *\end{array}$ & $\begin{array}{c}0.217 \\
* *\end{array}$ & $\begin{array}{c}0.091 \\
\text { ns }\end{array}$ & $\begin{array}{c}0.395 \\
* *\end{array}$ & $\begin{array}{c}0.050 \\
\mathrm{~ns}\end{array}$ & $\begin{array}{c}0.076 \\
\mathrm{~ns}\end{array}$ & $\begin{array}{c}0.085 \\
n s\end{array}$ & $\begin{array}{c}0.224 \\
* *\end{array}$ & $\begin{array}{c}0.045 \\
\text { ns }\end{array}$ & $\begin{array}{c}0.103 \\
\text { ns }\end{array}$ & $\begin{array}{c}0.024 \\
\mathrm{~ns}\end{array}$ & $\begin{array}{c}0.056 \\
\text { ns }\end{array}$ & $\begin{array}{c}0.042 \\
\text { ns }\end{array}$ & $\begin{array}{c}0.023 \\
\text { ns }\end{array}$ & $\begin{array}{c}-0.008 \\
\text { ns }\end{array}$ & $\begin{array}{c}-0.013 \\
n s\end{array}$ \\
\hline LWi & $\mathrm{rG}$ & & 1 & $\begin{array}{c}0.370 \\
* *\end{array}$ & $\begin{array}{c}0.108 \\
\text { ns }\end{array}$ & $\begin{array}{c}0.705 \\
* *\end{array}$ & $\begin{array}{c}0.573 \\
* *\end{array}$ & $\begin{array}{c}0.092 \\
\text { ns }\end{array}$ & $\begin{array}{c}0.244 \\
* *\end{array}$ & $\begin{array}{c}0.349 \\
* *\end{array}$ & $\begin{array}{c}-0.124 \\
*\end{array}$ & $\begin{array}{c}0.534 \\
* *\end{array}$ & $\begin{array}{c}-0.076 \\
n s\end{array}$ & $\begin{array}{c}0.167 \\
* *\end{array}$ & $\begin{array}{c}0.056 \\
\text { ns }\end{array}$ & $\begin{array}{c}-0.095 \\
\text { ns }\end{array}$ & $\begin{array}{c}-0.487 \\
* *\end{array}$ & $\begin{array}{c}-0.309 \\
* *\end{array}$ \\
\hline LWi & $\mathrm{rP}$ & & 1 & $\begin{array}{c}0.228 \\
* *\end{array}$ & $\begin{array}{c}0.061 \\
\text { ns }\end{array}$ & $\begin{array}{c}0.242 \\
* *\end{array}$ & $\begin{array}{c}0.096 \\
n s\end{array}$ & $\begin{array}{c}0.052 \\
\text { ns }\end{array}$ & $\begin{array}{c}0.151 \\
* *\end{array}$ & $\begin{array}{c}0.232 \\
* *\end{array}$ & $\begin{array}{c}-0.046 \\
\text { ns }\end{array}$ & $\begin{array}{c}0.175 \\
* *\end{array}$ & $\begin{array}{c}-0.043 \\
\text { ns }\end{array}$ & $\begin{array}{c}0.063 \\
\text { ns }\end{array}$ & $\begin{array}{c}0.011 \\
\mathrm{~ns}\end{array}$ & $\begin{array}{c}-0.016 \\
\text { ns }\end{array}$ & $\begin{array}{c}0.046 \\
\text { ns }\end{array}$ & $\begin{array}{c}-0.222 \\
* *\end{array}$ \\
\hline LWe & $\mathrm{rG}$ & & & 1 & $\begin{array}{c}0.190 \\
* *\end{array}$ & $\begin{array}{c}-0.188 \\
* *\end{array}$ & $\begin{array}{c}0.142 \\
*\end{array}$ & $\begin{array}{c}-0.074 \\
\text { ns }\end{array}$ & $\begin{array}{c}-0.206 \\
* *\end{array}$ & $\begin{array}{c}-0.278 \\
* *\end{array}$ & $\begin{array}{c}-0.147 \\
*\end{array}$ & $\begin{array}{c}0.235 \\
* *\end{array}$ & $\begin{array}{c}-0.659 \\
* *\end{array}$ & $\begin{array}{c}0.077 \\
\text { ns }\end{array}$ & $\begin{array}{c}0.241 \\
* *\end{array}$ & $\begin{array}{c}0.060 \\
\mathrm{~ns}\end{array}$ & $\begin{array}{c}0.575 \\
* *\end{array}$ & $\begin{array}{c}-0.671 \\
* *\end{array}$ \\
\hline LWe & $\mathrm{rP}$ & & & 1 & $\begin{array}{c}0.103 \\
\text { ns }\end{array}$ & $\begin{array}{c}-0.057 \\
\text { ns }\end{array}$ & $\begin{array}{c}0.047 \\
\text { ns }\end{array}$ & $\begin{array}{c}-0.028 \\
\text { ns }\end{array}$ & $\begin{array}{c}-0.122 \\
*\end{array}$ & $\begin{array}{c}-0.155 \\
* *\end{array}$ & $\begin{array}{c}-0.078 \\
\text { ns }\end{array}$ & $\begin{array}{c}0.014 \\
\text { ns }\end{array}$ & $\begin{array}{c}-0.200 \\
* *\end{array}$ & $\begin{array}{c}0.051 \\
\text { ns }\end{array}$ & $\begin{array}{c}0.020 \\
\text { ns }\end{array}$ & $\begin{array}{c}0.030 \\
\mathrm{~ns}\end{array}$ & $\begin{array}{c}0.024 \\
\mathrm{~ns}\end{array}$ & $\begin{array}{c}-0.491 \\
* *\end{array}$ \\
\hline HFW & $\mathrm{rG}$ & & & & 1 & $\begin{array}{c}0.168 \\
* *\end{array}$ & $\begin{array}{c}-0.380 \\
* *\end{array}$ & $\begin{array}{c}0.457 \\
* *\end{array}$ & $\begin{array}{c}0.079 \\
\mathrm{~ns}\end{array}$ & $\begin{array}{c}0.550 \\
* *\end{array}$ & $\begin{array}{c}0.204 \\
* *\end{array}$ & $\begin{array}{c}0.798 \\
* *\end{array}$ & $\begin{array}{c}0.712 \\
* *\end{array}$ & $\begin{array}{c}0.967 \\
* *\end{array}$ & $\begin{array}{c}0.610 \\
* *\end{array}$ & $\begin{array}{c}0.404 \\
* *\end{array}$ & $\begin{array}{c}0.433 \\
* *\end{array}$ & $\begin{array}{c}0.222 \\
* *\end{array}$ \\
\hline HFW & $\mathrm{rP}$ & & & & 1 & $\begin{array}{c}0.041 \\
\text { ns }\end{array}$ & $\begin{array}{c}-0.062 \\
\mathrm{~ns}\end{array}$ & $\begin{array}{c}0.132 \\
*\end{array}$ & $\begin{array}{c}0.067 \\
n s\end{array}$ & $\begin{array}{c}0.377 \\
* *\end{array}$ & $\begin{array}{c}0.111 \\
\text { ns }\end{array}$ & $\begin{array}{c}0.235 \\
* * \\
\end{array}$ & $\begin{array}{c}0.170 \\
* *\end{array}$ & $\begin{array}{c}0.452 \\
* *\end{array}$ & $\begin{array}{c}0.218 \\
* *\end{array}$ & $\begin{array}{c}0.130 \\
* *\end{array}$ & $\begin{array}{c}-0.025 \\
\text { ns }\end{array}$ & $\begin{array}{c}0.159 \\
* *\end{array}$ \\
\hline
\end{tabular}


Table 4. Genetic $\left(\mathrm{r}_{\mathrm{G}}\right)$ and phenotypic $\left(\mathrm{r}_{\mathrm{p}}\right)$ correlation coefficient (continued)

\begin{tabular}{|c|c|c|c|c|c|c|c|c|c|c|c|c|c|c|c|c|c|c|}
\hline & & LL & LWi & LWe & HFW & FL & FD & MT & $\mathrm{MpH}$ & HPW & PL & PWi & PT & HBW & $\mathrm{BL}$ & BWi & BT & CBBI \\
\hline FL & $\mathrm{rG}$ & & & & & 1 & $\begin{array}{c}0.507 \\
* *\end{array}$ & $\begin{array}{c}0.032 \\
\mathrm{~ns}\end{array}$ & $\begin{array}{c}0.256 \\
* *\end{array}$ & $\begin{array}{c}0.457 \\
* *\end{array}$ & $\begin{array}{c}0.266 \\
* *\end{array}$ & $\begin{array}{c}0.640 \\
* *\end{array}$ & $\begin{array}{c}0.747 \\
* *\end{array}$ & $\begin{array}{c}0.376 \\
* *\end{array}$ & $\begin{array}{c}0.472 \\
* *\end{array}$ & $\begin{array}{c}0.101 \\
\text { ns }\end{array}$ & $\begin{array}{c}-0.473 \\
* *\end{array}$ & $\begin{array}{c}0.176 \\
* *\end{array}$ \\
\hline FL & $\mathrm{rP}$ & & & & & 1 & $\begin{array}{c}0.162 \\
* *\end{array}$ & $\begin{array}{c}0.059 \\
\text { ns }\end{array}$ & $\begin{array}{c}0.066 \\
\text { ns }\end{array}$ & $\begin{array}{c}0.205 \\
* *\end{array}$ & $\begin{array}{c}0.086 \\
\text { ns }\end{array}$ & $\begin{array}{c}0.126 \\
*\end{array}$ & $\begin{array}{c}0.034 \\
\text { ns }\end{array}$ & $\begin{array}{c}0.085 \\
\text { ns }\end{array}$ & $\begin{array}{c}0.063 \\
\text { ns }\end{array}$ & $\begin{array}{c}0.093 \\
\text { ns }\end{array}$ & $\begin{array}{c}0.066 \\
\text { ns }\end{array}$ & $\begin{array}{c}0.073 \\
\text { ns }\end{array}$ \\
\hline FD & $\mathrm{rG}$ & & & & & & 1 & $\begin{array}{c}-0.280 \\
* *\end{array}$ & $\begin{array}{c}0.139 \\
*\end{array}$ & $\underset{* *}{0.172}$ & $\begin{array}{c}-0.085 \\
\text { ns }\end{array}$ & $\begin{array}{c}-0.320 \\
* *\end{array}$ & $\begin{array}{c}-0.332 \\
* *\end{array}$ & $\begin{array}{c}-0.513 \\
* *\end{array}$ & $\begin{array}{c}0.622 \\
* *\end{array}$ & $\begin{array}{c}-0.210 \\
* *\end{array}$ & $\begin{array}{c}-0.196 \\
* *\end{array}$ & $\begin{array}{c}-0.320 \\
* *\end{array}$ \\
\hline FD & $\mathrm{rP}$ & & & & & & 1 & $\begin{array}{c}-0.103 \\
\text { ns }\end{array}$ & $\begin{array}{c}0.030 \\
n s\end{array}$ & $\begin{array}{c}0.041 \\
n s\end{array}$ & $\begin{array}{c}-0.160 \\
* *\end{array}$ & $\begin{array}{c}-0.101 \\
\text { ns }\end{array}$ & $\begin{array}{c}-0.029 \\
\text { ns }\end{array}$ & $\begin{array}{c}-0.061 \\
n s\end{array}$ & $\begin{array}{c}0.108 \\
\text { ns }\end{array}$ & $\begin{array}{c}0.037 \\
n s\end{array}$ & $\begin{array}{c}0.155 \\
* *\end{array}$ & $\begin{array}{c}-0.101 \\
n s\end{array}$ \\
\hline MT & $\mathrm{rG}$ & & & & & & & 1 & $\begin{array}{c}0.353 \\
* *\end{array}$ & $\begin{array}{c}0.566 \\
* *\end{array}$ & $\begin{array}{c}-0.786 \\
* *\end{array}$ & $\begin{array}{c}0.014 \\
\text { ns }\end{array}$ & $\begin{array}{c}0.519 \\
* *\end{array}$ & $\begin{array}{c}0.520 \\
* *\end{array}$ & $\begin{array}{c}-0.067 \\
\text { ns }\end{array}$ & $\begin{array}{c}-0.036 \\
n s\end{array}$ & $\begin{array}{c}0.105 \\
\text { ns }\end{array}$ & $\begin{array}{c}0.160 \\
* *\end{array}$ \\
\hline MT & $\mathrm{rP}$ & & & & & & & 1 & $\begin{array}{c}0.105 \\
\text { ns }\end{array}$ & $\begin{array}{c}0.261 \\
* *\end{array}$ & $\begin{array}{c}-0.155 \\
* *\end{array}$ & $\begin{array}{c}0.027 \\
\text { ns }\end{array}$ & $\begin{array}{c}0.008 \\
\text { ns }\end{array}$ & $\begin{array}{c}0.115 \\
*\end{array}$ & $\begin{array}{c}0.002 \\
\text { ns }\end{array}$ & $\begin{array}{c}0.007 \\
\text { ns }\end{array}$ & $\begin{array}{c}0.018 \\
\text { ns }\end{array}$ & $\begin{array}{c}0.070 \\
\text { ns }\end{array}$ \\
\hline $\mathrm{MpH}$ & $\mathrm{rG}$ & & & & & & & & 1 & $\begin{array}{c}0.374 \\
* *\end{array}$ & $\begin{array}{c}-0.470 \\
* *\end{array}$ & $\begin{array}{c}-0.435 \\
* *\end{array}$ & $\begin{array}{c}-0.544 \\
* *\end{array}$ & $\begin{array}{c}-0.089 \\
\text { ns }\end{array}$ & $\begin{array}{c}0.038 \\
\text { ns }\end{array}$ & $\begin{array}{c}-0.026 \\
n s\end{array}$ & $\begin{array}{c}-0.845 \\
* *\end{array}$ & $\begin{array}{c}0.134 \\
*\end{array}$ \\
\hline $\mathrm{MpH}$ & $\mathrm{rP}$ & & & & & & & & 1 & $\begin{array}{c}0.236 \\
* *\end{array}$ & $\begin{array}{c}-0.251 \\
* *\end{array}$ & $\begin{array}{c}-0.137 \\
*\end{array}$ & $\begin{array}{c}-0.112 \\
\mathrm{~ns}\end{array}$ & $\begin{array}{c}-0.015 \\
\text { ns }\end{array}$ & $\begin{array}{c}0.014 \\
\text { ns }\end{array}$ & $\begin{array}{c}0.059 \\
\text { ns }\end{array}$ & $\begin{array}{c}-0.069 \\
\text { ns }\end{array}$ & $\begin{array}{c}0.130 \\
*\end{array}$ \\
\hline HPW & $\mathrm{rG}$ & & & & & & & & & 1 & $\begin{array}{c}-0.109 \\
\text { ns }\end{array}$ & $\begin{array}{c}0.392 \\
* *\end{array}$ & $\begin{array}{c}0.969 \\
* *\end{array}$ & $\begin{array}{c}0.382 \\
* *\end{array}$ & $\begin{array}{c}0.275 \\
* *\end{array}$ & $\begin{array}{c}0.000 \\
\mathrm{~ns}\end{array}$ & $\begin{array}{c}0.364 \\
* *\end{array}$ & $\begin{array}{c}0.357 \\
* *\end{array}$ \\
\hline HPW & $\mathrm{rP}$ & & & & & & & & & 1 & $\begin{array}{c}-0.071 \\
\mathrm{~ns}\end{array}$ & $\begin{array}{c}0.186 \\
*\end{array}$ & $\begin{array}{c}0.216 \\
* *\end{array}$ & $\underset{* *}{0.211}$ & $\begin{array}{c}0.016 \\
\text { ns }\end{array}$ & $\begin{array}{c}0.038 \\
\text { ns }\end{array}$ & $\begin{array}{c}0.077 \\
\text { ns }\end{array}$ & $\begin{array}{c}0.272 \\
* *\end{array}$ \\
\hline PL & $\mathrm{rG}$ & & & & & & & & & & 1 & $\begin{array}{c}0.472 \\
* *\end{array}$ & $\begin{array}{c}0.329 \\
* *\end{array}$ & $\begin{array}{c}0.286 \\
* *\end{array}$ & $\begin{array}{c}0.524 \\
* *\end{array}$ & $\begin{array}{c}0.557 \\
* *\end{array}$ & $\begin{array}{c}0.527 \\
* *\end{array}$ & $\begin{array}{c}0.159 \\
* *\end{array}$ \\
\hline PL & $\mathrm{rP}$ & & & & & & & & & & 1 & $\begin{array}{c}-0.024 \\
\text { ns }\end{array}$ & $\begin{array}{c}-0.009 \\
\text { ns }\end{array}$ & $\begin{array}{c}0.019 \\
\text { ns }\end{array}$ & $\begin{array}{c}0.226 \\
* *\end{array}$ & $\begin{array}{c}-0.050 \\
\text { ns }\end{array}$ & $\begin{array}{c}-0.011 \\
\text { ns }\end{array}$ & $\begin{array}{c}0.084 \\
\text { ns }\end{array}$ \\
\hline PWi & $\mathrm{rG}$ & & & & & & & & & & & 1 & $\begin{array}{c}0.398 \\
* *\end{array}$ & $\begin{array}{c}0.951 \\
* *\end{array}$ & $\begin{array}{c}0.229 \\
* *\end{array}$ & $\begin{array}{c}0.162 \\
*\end{array}$ & $\begin{array}{c}0.338 \\
* *\end{array}$ & $\begin{array}{c}0.668 \\
* *\end{array}$ \\
\hline PWi & $\mathrm{rP}$ & & & & & & & & & & & 1 & $\begin{array}{c}0.974 \\
* *\end{array}$ & $\begin{array}{c}0.172 \\
* *\end{array}$ & $\begin{array}{c}-0.04 \\
\text { ns }\end{array}$ & $\begin{array}{c}0.050 \\
\mathrm{~ns}\end{array}$ & $\begin{array}{c}0.045 \\
\text { ns }\end{array}$ & $\begin{array}{c}0.126 \\
*\end{array}$ \\
\hline PT & $\mathrm{rG}$ & & & & & & & & & & & & 1 & $\underset{* *}{0.951}$ & $\begin{array}{c}0.229 \\
* *\end{array}$ & $\begin{array}{c}-0.087 \\
\text { ns }\end{array}$ & $\begin{array}{c}0.162 \\
\text { ns }\end{array}$ & $\begin{array}{c}0.668 \\
* *\end{array}$ \\
\hline PT & $\mathrm{rP}$ & & & & & & & & & & & & 1 & $\begin{array}{c}0.172 \\
* *\end{array}$ & $\begin{array}{c}-0.004 \\
\text { ns }\end{array}$ & $\begin{array}{c}-0.089 \\
\text { ns }\end{array}$ & $\begin{array}{c}0.050 \\
\mathrm{~ns}\end{array}$ & $\begin{array}{c}0.126 \\
*\end{array}$ \\
\hline HBW & $\mathrm{rG}$ & & & & & & & & & & & & & 1 & $\begin{array}{c}0.502 \\
* *\end{array}$ & $\begin{array}{c}0.116 \\
*\end{array}$ & $\begin{array}{c}0.344 \\
* *\end{array}$ & $\begin{array}{c}0.146 \\
*\end{array}$ \\
\hline HBW & $\mathrm{rP}$ & & & & & & & & & & & & & 1 & $\begin{array}{c}0.128 \\
*\end{array}$ & $\begin{array}{c}0.276 \\
* *\end{array}$ & $\underset{* *}{0.188}$ & $\begin{array}{c}0.069 \\
\text { ns }\end{array}$ \\
\hline BL & $\mathrm{rG}$ & & & & & & & & & & & & & & 1 & $\begin{array}{c}0.731 \\
* *\end{array}$ & $\begin{array}{c}0.385 \\
* *\end{array}$ & $\begin{array}{c}-0.175 \\
* *\end{array}$ \\
\hline BL & $\mathrm{rP}$ & & & & & & & & & & & & & & 1 & $\begin{array}{c}0.074 \\
\mathrm{~ns}\end{array}$ & $\begin{array}{c}0.024 \\
\text { ns }\end{array}$ & $\begin{array}{c}-0.109 \\
\text { ns }\end{array}$ \\
\hline BWi & $\mathrm{rG}$ & & & & & & & & & & & & & & & 1 & $\begin{array}{c}0.064 \\
\text { ns }\end{array}$ & $\begin{array}{c}0.071 \\
\text { ns }\end{array}$ \\
\hline BWi & $\mathrm{rP}$ & & & & & & & & & & & & & & & 1 & $\begin{array}{c}0.237 \\
* *\end{array}$ & $\begin{array}{c}0.025 \\
\text { ns }\end{array}$ \\
\hline BT & $\mathrm{rG}$ & & & & & & & & & & & & & & & & 1 & $\begin{array}{c}0.127 \\
*\end{array}$ \\
\hline BT & $\mathrm{rP}$ & & & & & & & & & & & & & & & & 1 & $\begin{array}{c}0.011 \\
n s\end{array}$ \\
\hline
\end{tabular}

Note: Degree of freedom $=252, \mathrm{r}$ tabular at $\alpha 0.05=0.113, \mathrm{~ns}=$ not significant, $*=$ significant at $\alpha=0.05, \mathrm{r}$ tabular $\alpha 0.01=0.148, * *=$ highly significant at $\alpha 0.01 ; \mathrm{PH}=$ plant height $(\mathrm{m}), \mathrm{LL}=$ leaf length $(\mathrm{cm}), \mathrm{LWi}=$ leaf width $(\mathrm{cm}), \mathrm{LWe}=$ leaf weight $(\mathrm{g}), \mathrm{HFW}=100$ fruits weight $(\mathrm{g}), \mathrm{FL}=$ fruit length $(\mathrm{cm}), \mathrm{FD}=$ fruit diameter $(\mathrm{cm}), \mathrm{MT}=$ mesocarp thickness $(\mathrm{cm}), \mathrm{MpH}=$ mesocarp $\mathrm{pH}, \mathrm{HPW}=$ 100 parchments weight $(\mathrm{g}), \mathrm{PL}=$ parchment length $(\mathrm{cm}), \mathrm{PT}=$ parchment thickness $(\mathrm{cm}), \mathrm{HBW}=100$ beans weight $(\mathrm{g}), \mathrm{BL}=$ bean length $(\mathrm{cm}), \mathrm{BWi}=$ bean width $(\mathrm{cm}), \mathrm{CBBI}=$ coffee berry borer infestation $(\%)$

\section{CONCLUSION}

This research revealed morphological and genetic variation of the genotypes of Arabica coffee. The genotypes morphologically separated in three clusters based on the research locations. Leaf length, leaf width and leaf weight, hundred fruit weight, mesocarp thickness, mesocarp
$\mathrm{pH}$ and hundred parchment weight showed moderate genetic variation. Plant height, fruit length, fruit diameter, parchment length, parchment width, parchment thickness, hundred bean weight, bean length, bean width and bean thickness had low genetic variation. Because leaf weight had significant negative genetic correlation with coffee berry borer infestation, selection for higher leaf weight 
would be the best selection criterion to improve resistance of coffee against coffee berry borer. In future research, it could be needed to examine how $\mathrm{pH}$ of mesocarp could affect CBBI. Coffee hybridization is needed to obtain broad genetic diversity and big genetic advance.

\section{ACKNOWLEDGEMENT}

The authors thank the Research Institute of Nommensen HKBP University for the financial support with grant number 94/LPPM/IV/2014. We thank Prof. Dr. Muhamad Syukur, SP, MSi (Bogor Agriculture University) and Dr. Ir. Surip Mawardi (Indonesian Coffee and Cocoa Research Institute) for having given some advice for this manuscript.

\section{REFERENCES}

Aerts, R., G. Berecha, P. Gijbels, K. Hundera, S. Glabeke, K. Vandepitte, B. Muys, I. Roldan-Ruiz, O. Honnay. 2013. Genetic variation and risks of introgression in the wild Coffea arabica gene pool in South-Western Ethiopian montane rainforests. Evol. Appl. 6:243252. DOI: $10.1111 / \mathrm{j} .1752-4571.2012 .00285 . x$.

Atinafu, G., H. Mohammed, T. Kufa. 2017. Genetic variability of Sidama coffee (Coffea arabica L.) landrace for agro-morphological traits at Awada, Southern Ethiopia. Acad. Res. J. Agri. Sci. Res. 5: 263-275. DOI: 10.14662/ARJASR2017.025.

Beksisa, L., A. Ayano. 2016. Genetic variability, heritability and genetic advance for yield and yield components of Limmu coffee (Coffea arabica L.) accessions in South Western Ethiopia. Middle-East J. Sci. Res. 24:19131919. DOI: 10.5829/idosi.mejsr.2016.24.06.23518.

Constantin, M., Sobir, M. Syukur, W.B. Suwarno. 2017. Performance, heritability and genetic advance for oil yield and some economical characters in oil palm (Elaeis guineensis Jacquin) of Cameroon. J. Agron. Indonesia 45:212-219. DOI: http://dx.doi. org/10.24831/jai.v45i2.14110.

[DGEC] Directorate General of Estate Crops. 2017. Statistik Perkebunan Indonesia 2016-2018, Kopi. Dirjen Perkebunan, Jakarta.

Dani, N.K. Izzah, E. Randriani. 2016. Genetic variation within population of yellow-berried Arabica coffee cultivars at farmers field based on SSRs markers. J. TIDP 3:83-94. DOI: 10.21082/jtidp.v3n2.2016.

Fatimah, Urnemi, A.Z. Mustopa, H. Syahrumsyah. 2014. Molecular marker application of cherry and green bean of East Kalimantan coffee. Berita Biologi 1:1322.
Filho, O.G., P. Mazzafera. 2003. Caffeine and resistance of coffee to the berry borer Hypothenemus hampei (Coleoptera: Scolytidae). J. Agric. Food Chem 51: 6987-6991. DOI: 10.1021/jf0347968.

Geleta, M., I. Herrera, A. Monzon, T. Bryngelsson. 2012. Genetic diversity of Arabica coffee (Coffea arabica L.) in Nicaragua as estimated by simple sequence repeat markers. Sci. World J. 2012:1-11. DOI:1100/2012/939829.

Gessese, M.K., B. Bellachew, M. Jarso. 2015. Multivariate analysis of phenotypic diversity in the South Ethiopian coffee (Coffea arabica L.) for quantitative traits. Adv. Crop Sci. Tech. S1:003: 1-4. DOI:10.4172/23298863.S1-003.

Gichimu, B.M., C.O. Omondi. 2010. Morphological characterization of five newly developed lines of Arabica coffee as compared to commercial cultivars in Kenya. Int. J. Plant Breed. Genet. 4:238-246. DOI:10.3923/ijpbg.2010.238.246.

Green, P.W.C., A.P. Davis, A.A. Cosse, E. Vega. 2015. Can coffee chemical compounds and insecticidal plants be harnessed for control of major coffee pests? J. Agric. Food Chem. 63:9427-9434. DOI: 10.1021/ acs.jafc.5b03914.

Hanisch, S., Z. Dara, K. Brinkmann, A. Buerkert. 2011. Soil fertility and nutrient status of traditional Gayo coffee agroforestry systems in the Takengon region, Aceh Province, Indonesia. J. Agr. Rural Develop. Trop. Subtrop. 112:87-100.

Kathurima, C.W., G.M. Kenji, S.M. Muhoho, R. Boulanger, B.M. Gichimu, E.K. Gichuru. 2012. Genetic diversity among commercial coffee varieties, advanced selections and museum collections in Kenya using molecular markers. Int. J. Biodiv. Conserv. 2: 3946. DOI: $10.5897 / \mathrm{IJBC} 11.231$.

Kitila, O., S. Alamerew, T. Kufa, W. Garedew. 2011. Variability of quantitative traits in limmu coffee (Coffea arabica L.) in Ethiopia. Int. J. Agr. Res. 6:482-493. DOI:10.3923/ijar.2011.482.493.

Mishra, M.K., A. Slater. 2012. Recent advances in the genetic transformation of coffee. Biotech. Res. Int. 2012:1-17. DOI:10.1155/2012/580857.

Mayo, O. 1987. The Theory of Plant Breeding. Oxford University Press. New York. 
Oliveira, A.C.B., A.A. Pereira, F.L. Silva, J.C. Rezende, C.E. Botelho, G.R. Carvalho. 2010. Prediction of genetic gains from selection in Arabica coffee progenies. Crop Breeding Appl. Biotechnol. 11: 106-113. DOI: http://dx.doi.org/10.1590/S198470332011000200002 .

Quinn, G.P., M.J. Keough. 2002. Experimental Design and Data Analysis for Biologists. Cambridge University Press. New York.

Randriani, E., Dani, I. Sulistiyorini, E. Wardiana. 2014. Identification of characters affecting green bean yield of Arabica coffee in Garut regency using sequential path analysis. J. TIDP 3:175-184. DOI: 10.21082/ jtidp.v1n3.2014.p175-184.

Rodrigues, W.P., H.D. Vieira,D.H.S.G.Barbosa, C. Vittorazzi. 2012. Growth and yield of Coffea arabica L. in Fluminense: 2nd harvest. Rev. Ceres Viçosa 59:809815. DOI:10.1590/S0034-737X2012000600011.

Schmitt, C.B., F. Senbeta, M. Denich, H. Preisinger, H.J. Boehmer. 2009. Wild coffee management and plant diversity in the montane rainforest of Southwestern Ethiopia. Afr. J. Ecol. 48:78-86. DOI:10.1111/j.13652028.2009.01084.x.

Setotaw, T.A., E.T. Caixeta, G.F. Pena, E.M. Zambolim, A.A. Pereira, N.S. Sakiyama. 2010. Breeding potential and genetic diversity of "Híbrido do Timor" coffee evaluated by molecular markers. Crop Breeding Appl. Biotechnol. 10:298-304. DOI: 10.1590/S198470332010000400003 .

Silvestrini, M., M.P. Maluf, M.B. Silvarolla, O. GuerreiroFilho, H.P. Medina-Filho, M.M.T. Vanini, A.S.
Oliveira, C. Gaspari-Pezzopane, L.C. Fazuoli. 2008. Genetic diversity of a coffea germplasm collection assessed by RAPD markers. Genet. Res. Crop Evol. 55:901-910. DOI:10.1007/s10722-007-9295-5.

Sudradjat, A. 2010. The mapping of the Oldeman and Schmidt-Ferguson climate classification as an effort to utilize climate resources in the management of natural resources in North Sumatra. Tesis. Universitas Sumatera Utara. Medan.

Tessema, A., S. Alamerew, T. Kufa, W. Garedew. 2011.Variability and association of quality and biochemical attributes in some promising Coffea arabica germplasm collections in Southwestern Ethiopia. Internat. J. Plant Breeding Genet. 5:302316. DOI: 10.3923/ijpbg.2011.302.316.

Vega, F.E., F. Infante, A. Castillo, J. Jaramillo. 2009. The coffee berry borer, Hypothenemus hampei (Ferrari) (Coleoptera: Curculionidae): A short review, with recent findings and future research directions. Terrestrial Arthropod Rev. 2:129-147. DOI 10.1163 /187498209X12525675906031.

Wahyudi, T., Pujiyanto, Misnawi. 2016. Kopi: sejarah, botani, proses produksi, pengolahan, produksi hilir, dan sistem kemitraan. Gadjah Mada University Press. Yogyakarta.

Yuan, C.Y., P. Wang, P.P. Chen, W.J. Xiao, C. Zhang, S. Hu, P. Zhou, H. P. Chang, Z. He, R. Hu, X. T. Lu, J. Z. Ye, X. H. Guo. 2015. Genetic diversity revealed by morphological traits and ISSR markers in 48 okras (Abelmoschus escullentus L.). Physiol Mol. Biol. Plants 3:359-364. DOI: 10.1007/s12298-015-0303-5 